\title{
BMJ Open Quality 'Golden Patient': A quality improvement project aiming to improve trauma theatre efficiency in the Royal Gwent Hospital
}

Thomas Key, Gavin Reid, Nicola Vannet, John Lloyd, David Burckett-St. Laurent

To cite: Key T, Reid G, Vannet N, et al. 'Golden Patient': A quality improvement project aiming to improve trauma theatre efficiency in the Royal Gwent Hospital. BMJ Open Quality 2019;8:e000515. doi:10.1136/ bmjoq-2018-000515

Received 28 August 2018 Revised 3 January 2019 Accepted 29 January 2019
Check for updates

(C) Author(s) (or their employer(s)) 2019. Re-use permitted under CC BY-NC. No commercial re-use. See rights and permissions. Published by BMJ.

Orthopaedics, Royal Gwent Hospital, Newport, UK

Correspondence to Mr Thomas Key; thomaskey88@gmail.com

\section{ABSTRACT}

The efficiency of trauma lists when compared with elective orthopaedic lists is a frustration of many orthopaedic departments. At the Royal Gwent Hospital, late start times affecting total operating capacity of the trauma list were recognised as a problem within the department. The design team aimed to improve the start time of the list with the introduction of the 'golden patient' initiative. A protocol was agreed between the orthopaedic, anaesthetic and theatre staff where a 'golden patient' was selected for preoperative anaesthetic assessment by 14:00 the day before surgery and sent for at 08:15 as the first case on the trauma list. Baseline data was collected over a month. Two Plan-Do-Study-Act (PDSA) cycles were completed, one on the month the 'golden patient' initiative was implemented and one 4 months after the change. All data was collected from the Operating Room Management Information Service theatre system for the trauma theatre at the Royal Gwent Hospital. Results demonstrated significant improvement in patient arrival time in the theatre suite; PDSA1 by $33 \mathrm{~min}(p \leq 0.001)$ and PDSA2 by $29 \mathrm{~min}(\mathrm{p} \leq 0.001)$ and an earlier start of the first procedure; PDSA1 by $19 \mathrm{~min}(\mathrm{p}=0.018)$ and PDSA2 by $26 \mathrm{~min}(p \leq 0.001)$. There was also increased mean operating time per list (PDSA1 +16 min and PDSA2 +33 $\mathrm{min}$ ), increased total case number (PDSA $1+20$ cases and PDSA2 + 36 cases) and reduced cancellations (PDSA1 -2 cases and PDSA - 5 cases) compared with our baseline data. We demonstrated that the introduction of a 'golden patient' to the trauma theatre list improved the start time and overall operating capacity for the trauma list. Continuing this project, we plan to introduce assessment of all patients with fractured neck of femur in a similar way to the 'golden patient' to continue improving trauma theatre efficiency and reduce case cancellations.

\section{PROBLEN}

The Royal Gwent Hospital is a large district general hospital located in Newport in South Wales. The Royal Gwent orthopaedic department is made up of 16 orthopaedic consultants and remains the only orthopaedic unit in Wales to staff a two-tier senior and junior orthopaedic registrar rota.

Orthopaedic service delivery is split over two sites in Newport (the Royal Gwent Hospital and St Woolos Hospital) with another elective theatre unit Ysbyty Ystrad Fawr in Ystrad Mynach. Trauma services are delivered primarily in the main theatre complex at the Royal Gwent Hospital where three dedicated laminar flow orthopaedic theatres provide capacity for both trauma and elective cases.

Afull-day trauma list runs daily from Monday to Saturday from 08:00 until 17:00, hours are reduced from 08:00 until 14:00 on a Sunday. The consultant surgeon is contracted from 07:30 until 18:00 to include preoperative and postoperative patient review and anaesthetic staff from 08:00 until 18:00 to include perioperative care. Scrub staff work in shift patterns to staff the list appropriately including theatre set-up time. The operating department practitioner (ODP) starts at 08:15 and it is their role to send for the first patient once anaesthetic, scrub and surgical teams are satisfied. The anaesthetic cover for trauma lists consists of a consultant and registrar on weekdays and an anaesthetic consultant for the weekend. Each trauma list has a dedicated orthopaedic consultant and registrar and any specialist trauma cases are allocated to a trauma list covered by an orthopaedic consultant with the appropriate subspecialist interest.

All pending inpatient and outpatient trauma cases are listed on a trauma board and it is the role of both the orthopaedic consultant on call and a dedicated registrar trauma fellow to finalise the trauma list for each day. When the trauma workload exceeds that of the single trauma theatre, elective cases from the other two orthopaedic theatres are often cancelled to accommodate increased trauma workload.

Trauma theatre inefficiency with late start times and patient cancellations (often when patients were deemed medically unfit on the day of surgery) were common and a frustration for all those involved in the delivery of trauma care. 
The aims of this quality improvement project (QIP) were to improve trauma theatre efficiency by improving the start time of the list and therefore increase the overall operating capacity of the trauma list.

\section{BACKGROUND}

Efficiency of the orthopaedic trauma theatre, where patients are admitted as an emergency, is often compared with the efficiency of elective orthopaedic theatres. Although the majority of trauma theatre procedures are routine for orthopaedic surgeons, the non-elective nature of trauma combined with an ageing and medically complex population are recognised problems precipitating inefficiency of the trauma list.

A common reason for operative time lost on the trauma list is a late start, and although multifactorial, it is often a delay in basic orthopaedic surgical workup and anaesthetic assessment of the patient that contributes to this problem. Elective patients go through a thorough preassessment clinic process for suitability of surgery. However, for emergency admissions, basic investigations such as haematology, biochemistry, group and save and ECG are the responsibility of the admitting take team. Anaesthetic assessment of these often frail and comorbid patients can be a challenging process given the short period of time allocated in the morning prior to the list.

Late start times and patient cancellations often contribute to underutilisation of trauma operating capacity. Every minute of trauma theatre time represents a cost of $£ 24.77$, therefore to extrapolate, a delay of 1 hour to the start of the trauma list costs a trust approximately $£ 1486.20{ }^{1}$ It is well recognised that delay to theatre, particularly for patients with fractured neck of femur (NOF), increases morbidity and mortality rates. ${ }^{23}$ Increasing trauma theatre efficiency is important for improving patient outcomes and for a National Health Service under increasing economic restraints. Late start times are a recognised problem for emergency lists across the surgical specialties. ${ }^{4}$ Factors that have demonstrated an improvement in theatre efficiency include direct consultant supervision, improved communication within the theatre team and financial incentives. ${ }^{4-6}$ One technique proven to improve list start times is the selection of a predetermined first patient. ${ }^{7-9}$ Our multidisciplinary team devised a protocol tailored to our orthopaedic trauma unit for introducing the concept of the 'golden patient' to improve list efficiency.

\section{MEASUREMENT}

All theatre data in the Royal Gwent including the demographics, timings, nursing notes and surgical notes are recorded in the Operating Room Management Information Service (ORMIS) by the surgical and scrub teams. We planned to collect these data over a month's period in January 2018 to establish the baseline measurement of trauma theatre efficiency.
The start time of the first case on the trauma list is multifactorial, as it relies on the surgical workup of the patient by the orthopaedic team, completion of the nursing checklists on the ward, patient assessment by the anaesthetic team, the patient being sent for by the theatre staff, porters transporting the patient to the theatre suite and the set-up of anaesthetic and surgical equipment. To assess our interventions, we captured the time the patient reached the operating suite and knife to skin start of the surgical procedure from the ORMIS system.

The operating capacity of the trauma list was hypothesised to improve with an earlier time for the first patient to reach the operating suite. Operating capacity was assessed by collecting data on the finish time of the list (both as dressing on at end of case and time out of theatre), the number of operative cases completed and the total operating time (from knife to skin to dressing on for each procedure) assessed from each trauma list. To ascertain the number of cancellations, we collected data on both the total number and the reasons for each 'on the day' cancellation from the trauma list.

Our baseline data from January 2018 highlighted common frustrations with regard to the start time of the trauma list scheduled to start from 08:00. Our data showed the mean time of the patient arriving in the surgical suite as 09:19 with a mean time for the start of surgical procedure being 10:08. The operative productivity of the trauma list was a total case number of 99 surgical procedures over the month's period, with a total of $5555 \mathrm{~min}$ of operating time and a mean of 179.19 min operating per list. The finish time of the list was on average 16:04 with the patient leaving theatre at 16:20. A total of 26 cases were cancelled from the trauma list on the day of surgery with nine cases failing anaesthetic assessment as the patients were deemed medically unfit for their procedure that day.

We planned to implement our 'golden patient' initiative and collect data for the months' period from the date of implementation to establish if our intervention had improved the outcomes outlined above. We then aimed to assess compliance, address any problems and recollect our data.

\section{DESIGN}

The design of this study was to implement a 'golden patient' initiative to the trauma list. The design team involved a consultant anaesthetist, orthopaedic ST3 registrar, the consultant orthopaedic trauma lead and consultant orthopaedic audit lead. Data were collected and analysed by the orthopaedic ST3 and an orthopaedic CT1.

To improve the late start time observed in our baseline data, a patient was highlighted as the 'golden patient' and was assessed according to the following procedure.

By 14:00 each day 7 days a week, it was the role of both the orthopaedic consultant in charge of the current trauma list and the on call registrar to highlight a patient 
to be first on the trauma list as the 'golden patient' for the following day. This patient must have been fully worked up for theatre with appropriate investigations, consent and marking for the procedure. In the department, consent and surgical marking were the responsibility of the admitting on call team. They were rechecked on the post-take ward round, again on the morning of surgery by the operating surgeon and formed part of both the ward pretheatre checklist and WHO checklist.

The 'golden patient's' details were given to the consultant anaesthetist for the current trauma list and the patient underwent an anaesthetic assessment by either the consultant or their trainee. Any issues highlighted by the anaesthetic team were corrected at the earliest opportunity in order for the surgery to proceed the following morning. In the case that the patient highlighted was deemed as medically unfit for the procedure, they underwent optimisation in accordance with advice from the anaesthetic team and an alternative golden patient was selected and assessed.

The ward staff were informed when a patient was selected as the 'golden patient' so as to ensure that the theatre checklist was complete for the following morning. The patient was listed and highlighted on ORMIS as the 'golden patient' to inform the scrub staff so surgical kit and prosthesis requirements could be set up appropriately for the following morning.

Both the on call orthopaedic consultant and registrar remain on call for a 24-hour period. The surgeon in charge of the following days' trauma list was informed of the 'golden patient' by the on call team. This handover was reciprocated by the anaesthetic team who left a completed anaesthetic chart in the trauma theatre and also, when necessary, a courtesy call to the next days' trauma anaesthetist. At the 08:00 post-take trauma meeting in main theatres, a formal handover of the 'golden patient' and surgical plan was again discussed by the on call team with the surgeon in charge of the list.

Each morning, the 'golden patient' on the trauma was sent for at 08:15 when the ODP for trauma started their shift. This was to ensure arrival of the patient in the holding area before delays occurred due to porters being engaged in collecting other theatres' patients. The formal WHO team brief for the list was then discussed between anaesthetic, surgical and scrub staff before the golden patient was transferred to the anaesthetic room. The preassessed nature of the 'golden patient' saved time for the anaesthetist and enabled a prompt team briefing.

\section{STRATEGY}

\section{Plan-Do-Study-Act cycle 1}

This 'golden patient' strategy was agreed by the anaesthetic department, orthopaedic departments, theatre staff and ward staff, so as to ensure that all those involved in the trauma list were aware of the change in procedure. The strategy was discussed in both the monthly audit meeting and also via email to all relevant parties. This ensured that both the anaesthetic consultants, orthopaedic consultants and trainees agreed on the patient being sent for on the morning of surgery after prior assessment by their colleagues the day before. It also ensured the correct equipment was available for the surgical case so that theatre set-up and equipment availability did not delay the start of the case.

Posters were put up in the trauma handover room and in both the trauma theatre and anaesthetic room. This reminded all those involved to ensure the golden patient was highlighted the day before by 14:00 and to ensure that the golden patient was sent for by 08:15.

\section{Plan-Do-Study-Act cycle 2}

The design team were pleased with the improvement seen in the first cycle but identified six occasions during the month where no golden patient had been identified. Three of these occurred over a weekend. To improve compliance with the 'golden patient' strategy it was agreed that, if during the trauma list, a 'golden patient' had not been identified (due to no pending trauma) then a patient could be highlighted for assessment out of hours by the Confidential Enquiry into Perioperative Deaths (CEPOD) emergency list anaesthetists. The on call orthopaedic registrar was identified as being responsible for ensuring that a 'golden patient' was selected. The 'golden patient' was then discussed with CEPOD for anaesthetic assessment and with theatre staff for listing on ORMIS. CEPOD anaesthetic assessment consisted of an anaesthetic consultant and trainee well equipped to dealing with trauma patients and was considered a reasonable and safe alternative for assessment of the 'golden patient' out of hours.

\section{DATA COLLECTION AND ANALYSIS}

For each trauma list, the patient's arrival time in the surgical suite, procedure start time, procedure end time and list finish time were recorded as time elapsed from the 08:00 scheduled list start time. Descriptive statistics and graphs were used to explore the data initially. Data was unpaired at the three time points (baseline, PlanDo-Study-Act (PDSA) 1 and PDSA2). Data at all time points measured was found to be normally distributed via normal plots and the Kolmogorov-Smirnov test (ie, $\mathrm{p}>0.05$ ). Post hoc tests (via Bonferroni and Tukey options in SPSS) explored differences for comparisons between specific pairs of time points (ie, baseline vs PDSA1 and baseline vs PDSA2). All analyses were carried out using SPSS V.25. The total operating time for each list, total case number, number of on-the-day case cancellations and any occasions where the golden patient did not remain first were also compared.

\section{RESULTS}

A complete data set was collected for the baseline month (January), PDSA1 (February) and PDSA2 (May) with all data collected from ORMIS. The data collected in these 
Table 1 Comparison of theatre timings

\begin{tabular}{|c|c|c|c|}
\hline & Baseline & PDSA1 & PDSA2 \\
\hline In suite mean (time) & 09:19 & $08: 46$ & 08:50 \\
\hline Mean difference (min) & & -34 & -29 \\
\hline$P$ value & & ${ }^{*} \mathrm{P} \leq 0.001$ & ${ }^{*} \mathrm{P} \leq 0.001$ \\
\hline Procedure start mean (time) & $10: 08$ & 09:49 & 09:42 \\
\hline SE & & 6.7 & 6.6 \\
\hline$P$ value & & ${ }^{*} \mathrm{P}=0.018$ & ${ }^{*} \mathrm{P} \leq 0.001$ \\
\hline Procedure end mean (time) & $16: 04$ & $16: 57$ & $16: 56$ \\
\hline Mean difference (min) & & +53 & +52 \\
\hline SE & & 26.8 & 26.1 \\
\hline Mean difference (min) & & +48 & +44 \\
\hline SE & & 26.4 & 25.7 \\
\hline$P$ value & & $P=0.169$ & $P=0.200$ \\
\hline
\end{tabular}

${ }^{*}$ P, statistical significance at the $95 \% \mathrm{Cl}$.

PDSA, Plan-Do-Study-Act.

two cycles showed both improvement in the surgical start time of the trauma list and also improved trauma operating capacity in total case number and total operating time.

\section{Theatre start time}

Statistically significant improvement was demonstrated in both the time the patient arrived in the suite and the procedure start time (table 1). Arrival in suite improved from baseline to PDSA1 by a mean of $-33 \min (\mathrm{p} \leq 0.001)$ and from baseline to PDSA2 by a mean of $-29 \mathrm{~min}$ $(p \leq 0.001)$. Procedure start time improved from baseline to PDSA1 by a mean of $-19 \min (p \leq 0.018)$ and from baseline to PDSA2 by a mean of $-26 \min (p \leq 0.001)$.

\section{Theatre finish time}

Improvement was seen in both the procedure end time and the time out of theatre but this was not statistically significant (table 1). Procedure end time improved from baseline to PDSA1 by a mean of $+53 \mathrm{~min}(\mathrm{p}=0.124)$ and from baseline to PDSA2 by a mean of $+52 \mathrm{~min}$ $(p=0.126)$. Time out of theatre improved from baseline to PDSA1 by a mean of $+48 \mathrm{~min}(\mathrm{p}=0.169)$ and from baseline to PDSA2 by a mean of $+44 \mathrm{~min}(\mathrm{p}=0.2)$.

\section{Total operating time}

As a result of starting the list earlier, an increase was seen in the average operating time per list (table 2). This improved by a mean of $16 \mathrm{~min}$ per list from baseline to PDSA1 $(p=0.591)$ and a mean of $33 \mathrm{~min}$ per list from baseline to PDSA2 $(p=0.104)$. There was an increase in total case number (baseline with 99 cases, PDSA1 with 119 cases and PDSA2 with 135 cases). Although operating time did not demonstrate statistical significance, we argue that every minute of extraoperative time is a significant improvement for a trauma list.

\section{'Golden Patient' and on-the-day cancellations}

In PDSA1 there were six occasions where no golden patient was highlighted but the changes in PDSA2 resulted in full compliance. There were no on-the-day case cancellations of the 'golden patient' from the trauma list, however, infrequently, new trauma patients admitted after 'golden patient' selection were prioritised on four lists in PDSA1

Table 2 Comparison of theatre productivity

\begin{tabular}{|c|c|c|c|}
\hline Data period & $\begin{array}{l}\text { List mean operating time } \\
\text { (min) }\end{array}$ & Total operating time (min) & Total case number \\
\hline Baseline & 179.19 & 5555 & 99 \\
\hline PDSA2 & 212.48 & 6587 & 135 \\
\hline
\end{tabular}

PDSA, Plan-Do-Study-Act. 
and seven lists in PDSA2. The total number of on-the-day case cancellations was reduced (baseline with 26, PDSA1 with 23 and PDSA2 with 21). Many of these cancellations were due to reasons such as a lack of trauma ward beds or no available trauma theatre capacity. However, the number of patients cancelled as judged anaesthetically unfit on the day of surgery decreased (baseline of 9 , PDSA1 of 3 and PDSA2 of 5).

\section{LIMITATIONS}

This QIP achieved the aims outlined by the design team of improving the start time of the theatre list and therefore increasing the total operating time and total case number. However, of note, the team noticed a later finish time in theatre and a decrease in 'on the day' cancellations. As such a number of lessons were learnt during the process.

\section{Selection of the 'Golden Patient'}

It was decided that the 'golden patient' should be selected by 14:00 the day before the trauma list by the consultant in charge of the list that day and this was confirmed and checked by the on call registrar. The on call orthopaedic registrar was responsible for ensuring the patient had a complete surgical workup, was marked on the trauma board and highlighted to the anaesthetic team, theatre staff and ward staff. In circumstances where patient selection did not occur within normal working hours, the CEPOD anaesthetic team were asked to review the 'golden patient'.

\section{Anaesthetic assessment of the "Golden Patient'}

It was decided that anaesthetic assessment should be completed by the trauma theatre anaesthetic team. After the first month of this process, there were occasions when the trauma anaesthetic team consisted of a consultant without trainee assistance. This was true for all weekend trauma lists. Therefore, the anaesthetic consultant often did not have time to assess the 'golden patient' for the next day. To improve this situation, changes were made to ensure anaesthetic assessment was completed for the 'golden patient' by one of two other means. First was to delegate this role to one of the anaesthetic consultants or trainees from one of the two other orthopaedic theatres in the unit. Second, the 'golden patient' could be reviewed by the CEPOD anaesthetic team, particularly when assessment was needed out of hours. Although patients still underwent thorough assessment, it was recognised that this put an increased workload on the CEPOD anaesthetic team.

\section{Ensuring the 'Golden Patient' remained first}

Commonly, the 'golden patient' selected was an NOF patient as this patient cohort is at increased risk of morbidity and mortality when the time period between injury and surgery is increased. ${ }^{3}$ In circumstances where there was no NOF patient awaiting theatre, it was often other common orthopaedic trauma patients such as those requiring ankle surgery which were selected. As the 'golden patient' was selected at 14:00, there were still 18 hours of the 24 on call period where patients requiring surgery could be added to the trauma list.

In the PDSA1 cycle, there were four occasions when the 'golden patient' was moved to second prioritising a patient requiring a cauda equina decompression, a washout of a septic arthritis, a fractured NOF and a manipulation under anaesthetic (MUA) of a paediatric fracture. There were no occasions where other reasons such as equipment availability, a patient being judged medically unfit, consent and surgical marking issues or radiographer availability resulted in change of the 'golden patient'. The four major orthopaedic emergencies (compartment syndrome, cauda equina syndrome, open fractures and septic arthritis) are time-dependent cases and so should be prioritised on the trauma list. The design team felt it was appropriate for the septic joint and the cauda equina decompression to proceed first. However, the fractured NOF and paediatric MUA could arguably have waited until second. For the second cycle PDSA2, seven 'golden patients' were delayed until second on the trauma list. With the exception of one case, all of these were again paediatric MUAs. The team have taken this as an area for future improvement of the 'golden patient' protocol in our department. Intriguingly the number of on-the-day elective case cancellations from the other two orthopaedic theatres increased from baseline of 1 , to PDSA1 of 3 and PDSA2 of 4 . We believe this represents part of the culture shift in our department, sparked by the 'golden patient' initiative, for prioritising trauma patients in main theatres wherever possible.

\section{CONCLUSION}

Through successful selection and workup of the 'golden patient' involving the anaesthetic, orthopaedic and theatre staff, we have brought about a change in culture involving the start time of our daily trauma list. This has proved highly effective.

This QIP demonstrates that selection and assessment of a 'golden patient' according to our department initiative successfully achieved our aims. We acknowledge that improved theatre start time has been demonstrated in a previous 'golden patient' QIP by Roberts et al..$^{9}$ However, in comparison, we have achieved statistically significant improvement in the start time of list, and demonstrated an increase in the operative productivity of the trauma list in accordance with our hypothesis. Of particular interest to us was the later finish time in theatre that was observed. Although this could not be directly linked to an earlier start time, the team attributed this to a change in culture to a more 'can do' attitude adopted by the trauma theatre. We believe that by the time gained at the start of the list often allowed for an additional patient to be sent for in the afternoon. This further improved the efficiency of our trauma list as it resulted in an increase in the total number of operations performed. 
Future direction will involve sustaining the 'golden patient' initiative within our department where success relies heavily on the expedited patient assessment the day before surgery. In addition, we believe that the modest improvement in our case cancellations observed in this study has scope for larger scale improvement in the next phase of our project.

We are implementing an expansion of this initiative to include assessment and optimisation of all NOF patients on the day of admission. Communication regarding these patients between both the orthopaedic and anaesthetic teams on the day of admission will ensure all NOF patients are worked up and optimised as per Association of Anaesthetists of Great Britain and Ireland guidelines to avoid cancellations and delays to their surgical procedure. ${ }^{10}$

Acknowledgements We acknowledge the orthopaedic and anaesthetic departments of the Royal Gwent Hospital in addition to the orthopaedic scrub staff for engaging with this quality improvement project to improve our trauma theatre efficiency. We thank Dr Damian Farnell for his assistance with statistical analysis.

Contributors TK: design team, implemented the project, data collection, data analysis, project write-up and submitted the paper (responsible for overall content). GR: data collection and analysis. NV: implemented the project and edited the paper. $\mathrm{JL}$ : design team and implemented the project. DBSL: design team, implemented the project and edited the paper.

Funding The authors have not declared a specific grant for this research from any funding agency in the public, commercial or not-for-profit sectors.

Competing interests None declared.

Patient consent for publication Not required.

Provenance and peer review Not commissioned; externally peer reviewed.
Open access This is an open access article distributed in accordance with the Creative Commons Attribution Non Commercial (CC BY-NC 4.0) license, which permits others to distribute, remix, adapt, build upon this work non-commercially, and license their derivative works on different terms, provided the original work is properly cited, appropriate credit is given, any changes made indicated, and the use is non-commercial. See: http://creativecommons.org/licenses/by-nc/4.0/.

\section{REFERENCES}

1. Ang WW, Sabharwal S, Johannsson $\mathrm{H}$, et al. The cost of trauma operating theatre inefficiency. Ann Med Surg 2016;7:24-9.

2. The National Hip Fracture Database. NHFD 2018 annual report. https://www.nhfd.co.uk (Accessed Jul 2018).

3. Morrissey N, Iliopoulos E, Osmani AW, et al. Neck of femur fractures in the elderly: does every hour to surgery count? Injury 2017;48:1155-8.

4. Mohan A, Lutterodt C, Leon-Villapalos J. Operating efficiency of an emergency Burns theatre: an eight month analysis. Burns 2017;43:1435-40.

5. Turnbull GS, Hakimi M, McLauchlan GJ. Trauma theatre productivity - does the individual surgeon, anaesthetist or consultant presence matter? Injury 2018;49:969-74.

6. Halim UA, Khan MA, Ali AM. Strategies to improve start time in the operating theatre: a systematic review. J Med Syst 2018;42:160.

7. Oakley B, Nightingale J, Moran CG, et al. Does achieving the best practice tariff improve outcomes in hip fracture patients? An observational cohort study. BMJ Open 2017;7:e014190.

8. Javed S, Peck C, Salthouse D, et al. A predetermined first patient on the trauma list can improve theatre start times. Injury 2013;44:1528-31.

9. Roberts S, Saithna A, Bethune R. Improving theatre efficiency and utilisation through early identification of trauma patients and enhanced communication between teams. BMJ Qual Improv Rep 2015;4:u206641.w2670.

10. The Association of Anaesthetists of Great Britain and Ireland. Management of proximal hip fractures AAGBI. 2012 https://www. aagbi.org/sites/default/files/femoral\%20fractures\%202012_0.pdf (Accessed Jul 2018). 